\title{
PARASITOSES INTESTINAIS
}

\section{EM CRIANÇAS DE CRECHE COMUNITÁRIA}

EM FLORIANÓPOLIS, SC, BRASIL

\author{
Juliano dos Santos ${ }^{1}$, Adriana Regina Meinchein Duarte', Grazziella Gadotti ${ }^{1}$ e \\ Lenilza Mattos Lima
}

\section{RESUMO}

No presente estudo, foi observada a frequência de enteroparasitoses em crianças de 2 a 6 anos de idade, matriculadas na Creche Municipal Anjo da Guarda, na cidade de Florianópolis, Santa Catarina, Brasil. Foram analisadas 57 amostras fecais de agosto a novembro de 2012 pelos métodos de Lutz e de Faust et al. Obteve-se positividade para enteroparasitos em 61,4\% das crianças, sendo encontrados os seguintes agentes etiológicos: Blastocystis hominis (40,4\%), Giardia lamblia (24,6\%), Entamoeba coli (22,8\%), Endolimax nana (12,3\%), Entamoeba histolytica/E. dispar (7,1\%), Entamoeba hartmanni, Enterobius vermicularis e Iodamoeba bütschlii (1,8\%). Em relação ao grau de parasitismo, 24,6\% das crianças apresentavam monoparasitismo, 26,3\% biparasitismo e $10,5 \%$ poliparasitismo. A faixa etária mais acometida foi a de 5 a 6 anos $(72,2 \%)$. Os resultados observados mostraram elevada prevalência de protozoários intestinais e reforçam a necessidade da implantação de medidas de prevenção e educação em saúde que visem melhorar as condições de vida das crianças e dos adultos.

DESCRITORES: Creche; parasitos intestinais; frequência.

\begin{abstract}
Intestinal parasites in children at a community creche in Florianópolis, SC, Brazil

In the present study the frequency of intestinal parasites was observed in a group of 2 to 6 year old children enrolled in Anjo da Guarda municipal daycare, in Florianópolis, Santa Catarina, Brazil. 57 fecal samples were processed from August to November of 2012, using the methods of Lutz and Faust et al.. $61.4 \%$ of the children tested positive for intestinal parasites. The etiological agents were: Blastocystis hominis (40.4\%), Giardia lamblia (24.6\%), Entamoeba coli (22.8\%), Endolimax nana (12.3\%), Entamoeba histolytica/Entamoeba dispar (7.1\%), Entamoeba hartmanni, Enterobius
\end{abstract}

1 Discente do curso de Farmácia, Análises Clínicas, Universidade Federal de Santa Catarina, Brasil.

2 Departamento de Análises Clínicas, Universidade Federal de Santa Catarina, Brasil

Endereço para correspondência: Lenilza Mattos Lima, Universidade Federal de Santa Catarina, Centro de Ciências da Saúde, Departamento de Análises Clínicas. Campus Universitário, Trindade, CEP 88040-970, Florianópolis, SC, Brasil. Caixa-postal: 476. E-mail: lenilza@ccs.ufsc.br

Recebido para publicação em: 16/9/2013. Revisto em: 26/6/2014. Aceito em: 21/8/2014. 
vermicularis and Iodamoeba bütschlii (1.8\%). With respect to the degree of parasitism, $24.6 \%$ of children had monoparasitism, $26.3 \%$ had biparasitism and $10.5 \%$ had polyparasitism. Children aged 5 to 6 years presented more parasites $(72.2 \%)$. The observed results showed a high prevalence of intestinal protozoa, and reinforce the importance of implementing preventative measures and health education to improve the lives of children and adults.

KEY WORDS: Daycare; intestinal parasites; frequency.

\section{INTRODUÇÃO}

As parasitoses intestinais ainda apresentam elevada prevalência entre a população de baixo nível socioeconômico, representando um importante problema de saúde pública, principalmente pelos efeitos que podem ocasionar sobre o estado nutricional e o desenvolvimento físico da população infantil $(2,8,12,31,39)$. As crianças estão mais suscetíveis às infecções e reinfecções por estarem mais expostas aos agentes etiológicos e também porque, quando realizam sua higiene pessoal sozinhas, esta pode não ser satisfatória $(16,31,39)$. A elevada prevalência de enteroparasitos tem sido considerada a principal causa de morbidade entre os escolares de países em desenvolvimento $(15,29)$. A elevada ocorrência de protozoários como Giardia lamblia, Entamoeba coli, Entamoeba histolytica/E. dispar e Blastocystis hominis tem como origem tanto a transmissão interpessoal entre as crianças quanto a ingestão de água e/ou alimentos contaminados e mal higienizados, fatores estes associados à carência de rede de esgoto e água $(4,6,15$, 28). A associação parasitária é comum, uma vez que a aquisição de diversos agentes etiológicos ocorre pelo mesmo mecanismo de transmissão $(27,30)$.

O espectro clínico da infecção por parasitos intestinais varia de assintomático a um quadro caracterizado por dor abdominal, cólicas, náuseas, vômitos, diarreia, anemia, emagrecimento, falta de apetite e quadros de doenças respiratórias $(23,27,37)$. Outros agravos que os parasitos intestinais podem causar incluem obstrução intestinal (Ascaris lumbricoides) e má absorção de nutrientes (E. histolytica/E. dispar e G. lamblia). As manifestações clínicas são usualmente proporcionais à carga parasitária albergada pelo indivíduo (27).

Nos dias atuais, em razão do número cada vez maior de mulheres que ingressam no mercado de trabalho, a creche é uma realidade na vida das famílias, sendo este o local onde muitas crianças passam a maior parte de sua infầncia. Esta instituição tem papel importante no desenvolvimento integral da criança em seus aspectos físico, psicológico, intelectual e social. Nas instituições escolares, alguns problemas de saúde podem ser detectados, uma vez que delas devem fazer parte a prevenção e a educação em saúde. As crianças que frequentam creches estão mais sujeitas a infecções do que aquelas que são mantidas em suas residências por causa do grande contato interpessoal propiciado pelos ambientes coletivos $(3,16,19,36)$.

Vários autores já mostraram percentuais relevantes na prevalência de enteroparasitos em crianças de creches de diversos locais e regiões do Brasil (10, 16, 
18, 21, 35, 36, 39). É importante ressaltar que as informações sobre enteroparasitoses servem como indicador das condições de saneamento em que vive uma determinada população e que os portadores assintomáticos são disseminadores da doença, portanto contaminam o meio ambiente. No município de Florianópolis-SC, esta situação vem sendo observada pela carência de investimentos em infraestrutura, saneamento e saúde, que reflete diretamente nas condições ambientais da cidade (9).

O presente estudo visou avaliar a ocorrência de parasitos intestinais em crianças matriculadas na Creche Municipal Anjo da Guarda, localizada no Morro da Penitenciária, em Florianópolis-SC.

\section{MATERIAL E MÉTODOS}

O censo do Instituto Brasileiro de Geografia e Estatística (IBGE), de 2010, classifica a comunidade do Morro da Penitenciária, em Florianópolis, SC, onde está localizada a creche Anjo da Guarda, como aglomerado subnormal (local com carência de serviços públicos essenciais como coleta de lixo, rede de esgoto, rede de água, energia elétrica e iluminação pública). Segundo o censo, a população total da comunidade é de 1.092 pessoas, sendo 528 do sexo masculino e 564 do sexo feminino. A média de habitantes por domicílio é de 3,6 pessoas. Esta comunidade é composta por famílias que migraram do Planalto Serrano e Oeste Catarinense e foram se instalando na encosta do morro sem infraestrutura e saneamento básico.

A presente pesquisa foi aprovada pelo Comitê de Ética em Pesquisa Humana da UFSC, cujo pareceré o de $\mathrm{n}^{\mathrm{o}} 128.527$. Foi realizada no período de agosto a novembro de 2012 e envolveu 57 crianças das 95 matriculadas com idades entre 2 e 6 anos. Primeiramente, foi realizada uma reunião com os pais e/ou responsáveis e professores quando foram apesentados os esclarecimentos sobre a importância do trabalho e oferecidas, em texto impresso, as orientações para a coleta de fezes. As amostras fecais (uma amostra de fezes de cada criança) foram coletadas em solução SAF (Acetato de sódio, ácido acético e formol a 10\%). As amostras foram analisadas pelo método de Lutz (22) e de Faust e colaboradores (14), no Laboratório Didático de Parasitologia Clínica do Departamento de Análises Clínicas/UFSC. Para as análises, utilizaram-se solução de Lugol e coloração tricrômica (11). Para cada amostra foi analisada uma lâmina por método e dois examinadores leram o mesmo material. Os resultados obtidos foram analisados no programa Excel 2010, sendo aplicado o teste qui-quadrado com nível de significância de 5\%.

Os resultados dos exames foram entregues aos pais ou responsáveis para encaminhamento médico.

\section{RESULTADOS}

Os resultados evidenciaram que 61,4\% (35/57) das amostras (Figura 1) apresentavam positividade para parasitos intestinais, os quais estão relacionados na 
Tabela 1. Observou-se que $B$. hominis foi o protozoário mais frequente, com índice de positividade de 40,4\% (23/57), seguido por G. lamblia com 24,6\% (14/57) e $E$. coli $\operatorname{com} 22,8 \%(13 / 57)$.

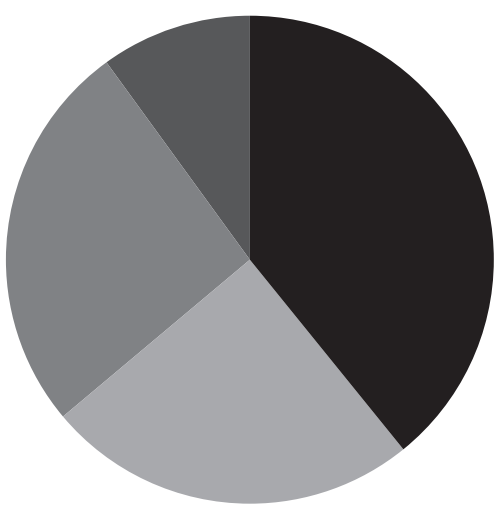

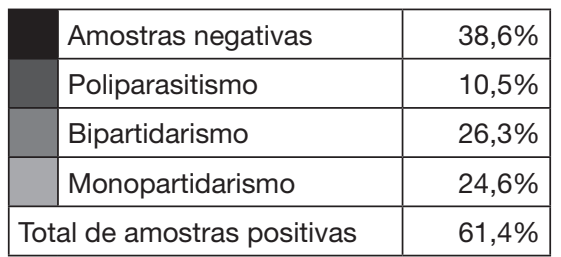

Figura 1. Prevalência (\%) de amostras fecais positivas e negativas segundo o grau de parasitismo encontrado nas 57 crianças da Creche Municipal Anjo da Guarda, em Florianópolis, SC, Brasil.

Tabela 1. Frequência de enteroparasitos em 57 amostras fecais de crianças da Creche Municipal Anjo da Guarda, Florianópolis-SC

\begin{tabular}{lc}
\hline \multicolumn{1}{c}{ Enteroparasito } & Frequência n(\%) \\
\hline Blastocystis hominis & $23(40,4)$ \\
\hline Giardia lamblia & $14(24,6)$ \\
\hline Entamoeba coli & $13(22,8)$ \\
\hline Endolimax nana & $7(12,3)$ \\
\hline Entamoeba histolytica/E. dispar & $4(7,0)$ \\
\hline Entamoeba hartmanni & $1(1,8)$ \\
\hline Enterobius vermicularis & $1(1,8)$ \\
\hline Iodamoeba bütschilii & $1(1,8)$ \\
\hline
\end{tabular}

Em relação ao parasitismo por helmintos, entre as 57 amostras analisadas, somente uma $(1,8 \%)$ tratava-se desse tipo de parasitismo e $35(61,4 \%)$ estavam parasitadas por protozoários. Estatisticamente houve diferença significativa $(\mathrm{p}<0,025)$.

Na Figura 2, observa-se que o gênero mais acometido por parasitoses foi o masculino (23/32), vindo em seguida o feminino (12/25), porém sem significância estatística $(\mathrm{p}>0,05)$. A faixa etária mais acometida por enteroparasitos nos escolares avaliados foi a de 5 a 6 anos (13/18), seguida pela faixa etária de 2 a 4 anos (22/39), também não havendo diferença estatística significativa $(p>0,05)$. 


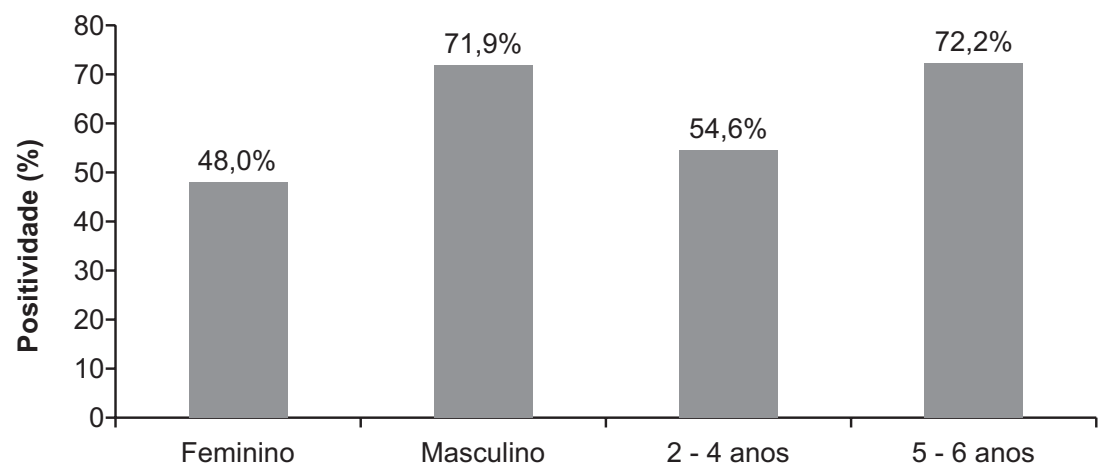

Figura 2. Frequência (\%) de positividade por gênero e faixa etária nas 57 amostras analisadas.

\section{DISCUSSÃO E CONCLUSÕES}

Em Florianópolis-SC, a Creche Municipal Anjo da Guarda está localizada numa região onde o acelerado crescimento demográfico não foi acompanhado pelo estabelecimento de saneamento básico e água potável em grande número de residências. Em adição, a higiene pessoal inadequada, o contato interpessoal, a ingestão de água e alimentos contaminados e o maior contato com o solo e animais aumentam a probabilidade de disseminação parasitária nas crianças.

A elevada frequência de $61,4 \%$ para enteroparasitos foi semelhante aos resultados obtidos em 2010 por Aguiar e Fachini (65,0\%) em Florianópolis, SC (1), em 2011 por Vasconcelos et al. (60,8\%) em Crato, CE (38), em 2009 por Costa et al. (65,5\%) em Maceió, AL (10) e por Fonseca e Silveira (56,5\%) em Anápolis, GO (17). Por outro lado, a positividade no presente estudo foi inferior a encontrada em 2006 por Teixeira et al. (73,3\%) em Concórdia, SC (35) e superior aos resultados obtidos em 2009 por Uchôa et al. (51,6\%) em Niterói, RJ (36) e, em 2008, por Zaiden et al. (39,9\%) em Rio Verde, GO (39).

Quanto ao grau de parasitismo, foi comum encontrar mais de um parasito nas amostras fecais, havendo o predomínio de biparasitismo. Outros autores relataram resultados superiores ao do presente estudo: Fonseca e Silveira (2009) verificaram 51\% de monoparasitismo e $49 \%$ de poliparasitismo (17); Aguiar e Fachini (2010) observaram 39\% de monoparasitismo, 29\% de biparasitismo e 32\% de poliparasitismo (1); Seixas et al. (2011) observaram que 39\% tinham apenas um parasito, $33 \%$ estavam biparasitados e $22 \%$ apresentaram poliparasitismo (30).

Em relação à frequência dos enteroparasitos, o protozoário $B$. hominis foi o mais encontrado, seguido por G. lamblia. Em Florianópolis-SC, Aguiar e Fachini (2010) encontraram índice inferior (16,1\%) para B. hominis (1); Pezzani et al. (2012), na Argentina, detectaram índice de 27,5\% para esse protozoário em crianças da 
zona suburbana e $11,9 \%$ da zona rural (26). A prevalência de $B$. hominis em regiões de um mesmo país pode variar amplamente e esse protozoário é encontrado mais frequentemente em estudos epidemiológicos. Por outro lado, a natureza pleomórfica do $B$. hominis e a falta de padronização nas técnicas para sua deteç̧ão têm gerado confusão e, em alguns casos, levado a interpretações erradas dos resultados. A elevada prevalência de $B$. hominis tem sido associada com falta de higiene, exposição a animais e consumo de água e alimentos contaminados. Entretanto, ainda ocorrem divergências em relação ao potencial patogênico do B. hominis (34).

A giardíase tem sido alvo de pesquisas sobre síndromes diarreicas em crianças de creches $(19,25)$. A frequência encontrada para G. lamblia no presente estudo foi semelhante àquela verificada nos estudos de Zaiden et al. (21,4\%), em 2008, em Rio Verde, GO (39), e de Gonçalves et al. (19,2\%), em 2011, em Uberlândia, MG (18). Índices inferiores foram encontrados, em 2010, por Aguiar e Fachini (12,6\%) em Florianópolis, SC (1) e por Pezzani et al. (7,5\% e 9,6\%), em 2012, na Argentina (26). Resultados superiores foram verificadas por Fonseca e Silveira (41\%), em 2009, em Anápolis, GO (17) e por Costa et al. (38,4\%), em 2009, em Maceió, AL (10).

Neste estudo, o que mais chamou a atenção foi o grande número de crianças infectadas por G. lamblia e não infectadas para geohelmintos. No caso da giardíase, isso pode ser devido ao fato de que os cistos do protozoário são resistentes ao tratamento da água com cloro (7) e à transmissão interpessoal entre as crianças, pois os cistos são infectantes quando eliminados nas fezes (33).

O espectro clínico da amebíase causada por E. histolytica varia de assintomático a colite fulminante ou até mesmo abscessos hepáticos. A transmissão é fecal-oral, podendo ocorrer por meio da água poluída com dejetos humanos, da contaminação dos alimentos e das mãos sujas contendo os cistos do protozoário (27). $O$ índice de positividade identificado para E. histolytica/E. dispar neste estudo foi semelhante ao encontrado, em 2010, por Aguiar e Fachini $(8,0 \%) \mathrm{em}$ Florianópolis, SC (1). Fonseca e Silveira (17) em 2009, Seixas et al. (30) em 2011 e Santos et al. (28) em 2014 obtiveram resultados superiores: 15\% , 21,5\% e 25\%, respectivamente.

Os protozoários E. coli, E. nana, E. hartmanni e I. bütschlii são considerados parasitos comensais, porém apresentam grande importância no exame parasitológico de fezes. Eles indicam contaminação fecal do ambiente ao qual as crianças estão expostas, assim como possível contaminação da água e de alimentos, compartilhando as vias de transmissão dos parasitos patogênicos (7).

As diferentes frequências de enteroparasitoses verificadas nos estudos realizados por vários autores no Brasil podem ser devidas à dispersão regional desigual do agente etiológico e também às diferenças entre as sensibilidades das técnicas utilizadas para o diagnóstico.

Na presente pesquisa, houve um predomínio de protozoários em relação aos helmintos, observando-se apenas uma amostra positiva para E. vermicularis e 
nenhuma positividade para outros helmintos. Resultado semelhante foi encontrado por Mariz e Nóbrega (2009) em Campina Grande, PB, os quais relataram 65\% de protozoários (24). A baixa prevalência de helmintos pode ser justificada pela crescente utilização de medicamentos anti-helmínticos, principalmente os azólicos (5). É possível que os geohelmintos não estejam circulando na comunidade ou, ainda, que as crianças, pelo uso de calçados, estejam tendo menor contato com larvas de helmintos que infectam por via cutânea.

A baixa prevalência para E. vermicularis no presente estudo já era esperada, uma vez que o exame coproparasitológico não é suficientemente sensível para a detecção desse helminto, podendo esporadicamente apresentar-se positivo. $\mathrm{O}$ mais coerente seria utilizar o método de Graham ou fita adesiva (11).

Houve uma prevalência maior de amostras positivas em crianças do gênero masculino em comparação com crianças do gênero feminino. Quanto à faixa etária, a prevalência foi maior entre crianças de 5 a 6 anos comparativamente à faixa de 2 a 4 anos. A maior prevalência da infecção entre as crianças do gênero masculino pode estar relacionada ao fato de que os meninos ficam mais expostos ao ambiente peridomiciliar durante as atividades de lazer (13). Em relação à faixa etária, observa-se uma tendência ao aumento da positividade à medida que aumenta a idade das crianças. Este resultado pode ser devido ao fato de as crianças, na faixa etária mais elevada, possuírem maior autonomia, maior contato com o solo, o que favorece a contaminação por parasitos, inclusive com maior diversidade de espécies (21).

Diante dos resultados observados, conclui-se que há uma prevalência de parasitoses intestinais, principalmente de protozoários, em crianças da creche Anjo da Guarda. Ficaram evidenciadas, também, a importância da conscientização da população para a ingestão de água fervida ou filtrada e de alimentos bem lavados e a necessidade da melhoria das condições habitacionais com a instalação de sistemas de água tratada e redes de esgoto pelo município de Florianópolis, SC. Nas creches, é importante realizar ações educativas por meio de atividades lúdicas, nas quais seja abordada a transmissão e as medidas de prevenção das parasitoses (32).

\section{REFERÊNCIAS}

1. Aguiar MA, Fachini D. Prevalência de enteroparasitoses em escolares da comunidade da Serrinha, Florianópolis, SC. Florianópolis [Monografia de conclusão da Disciplina de Estágio Supervisionado em Análises Clínicas ACL/UFSC], 2010.

2. Andrade EC, Leite ICG, Rodrigues VO, Cesca MG. Parasitoses intestinais: uma revisão sobre seus aspectos sociais, epidemiológicos, clínicos e terapêuticos. Rev APS 13: 231-240, 2010.

3. Araujo Filho HB, Carmo-Rodrigues MS, Mello CS, Melli LCFL, Tahan S, Morais MB. Parasitoses intestinais se associam a menores índices de peso e estatura em escolares de baixo estrato econômico. Rev Paul Pediatr 29: 521-528, 2011.

4. Baptista AB, Ramos LS, Santos HAG. Prevalência de enteroparasitoses e aspectos epidemiológicos de crianças e jovens no município de Altamira-PA. Rev Pesq Saúde 14: 77-80, 2013.

5. Bethony JR, Brooker S, Albonico M, Geiger SM, Loukas A, Diemert D, Hotez PJ. Soil-transmitted helminth infections: ascariasis, trichuriasis, and hookworm. Lancet 367: 1521-1532, 2006. 
6. Bezerra FSM, Oliveira MF, Miranda ALL. Incidência de parasitos intestinais em material subungueal e fecal em crianças da Creche Aprisco, Fortaleza, CE. Rev Bras Anal Clin 35: 39-40, 2003.

7. Borges WF, Marciano FM, Oliveira HB. Parasitos intestinais: elevada prevalência de Giardia lamblia em pacientes atendidos pelo serviço público de saúde da região sudeste de Goiás, Brasil. Rev Patol Trop 40: 149-157, 2011.

8. Carvalho-Costa FA, Gonçalves AQ, Lassance SL, Silva Neto LM, Salmazo CAA, Bóia MN. Giardia lamblia and other intestinal parasitic infections and their relationships with nutritional status in children in Brazilian Amazon. Rev Inst Med Trop São Paulo 49: 147-153, 2007.

9. Conexões de saberes. Relatório de pesquisa na Serrinha - conhecendo melhor a comunidade. UFSC, 2007. Disponível em: http://www.conexoes.ufsc.br. Acesso em 30/05/2012.

10. Costa SS, Silva BFP, Morais AFC, Wanderley FS. Ocorrência de parasitas intestinais em material subungueal e fecal em crianças de uma creche no município de Maceió, Alagoas. Rev Pediatr 31: 198-203, 2009.

11. De Carli GA. Parasitologia Clínica: Seleção de técnicas e métodos de laboratório para o diagnóstico de parasitoses humanas. Atheneu. São Paulo, 2011.

12. Del Barco M, Hugo O, Paula MA, Raúl LI. Parasitosis intestinal. Rev FMC 16: 14-24, 2009.

13. Faleiros JM, Gallo G, Silva MM, Raful R, Nasorri AR, Pipino LF. Ocorrência de enteroparasitoses em alunos da escola pública de ensino fundamental do município de Catanduva (São Paulo, Brasil). Rev Inst Adolfo Lutz 63: 243-247, 2004.

14. Faust EC, D'Antoni JS, Odon V, Miller MJ, Perez C, Sawitz W, Thomen LF, Tobie J, Walker JH. A critical study of clinical laboratory technics of the diagnosis of protozoan cysts and helminth eggs in feces. I - Preliminary communication. Am J Trop Med Hyg 18: 169, 1938.

15. Ferreira H, Lala ERP, Monteiro MC, Raimondo ML. Estudo epidemiológico localizado da frequência e fatores de risco para enteroparasitoses e sua correlação com o estado nutricional de crianças em idade pré-escolar. Publ UEPG Ci Biol Saúde 12: 33-40, 2006.

16. Figueiredo MIO, Serres OS, Suterio GM, Mello MAM, Altermann GTM. Parasitoses intestinais em crianças com idade de 24 a 58 meses das escolas municipais de educação infantil, relacionando alguns aspectos socioeconômicos, Uruguaiana, RS. Rev NewsLab 106: 180-189, 2011.

17. Fonseca KCLE, Silveira LVP. Estudo das parasitoses gastrointestinais em crianças de 0 a 12 anos atendidas pelo laboratório central do município de Anápolis. Anuário da produção de iniciação cientifica discente 12: 77-95, 2009.

18. Gonçalves ALR, Belizário TL, Pimentel JB, Penatti MPA, Pedroso RS. Prevalence of intestinal parasites in preschool children in the region of Uberlândia, State of Minas Gerais, Brazil. Rev Soc Bras Med Trop 44: 191-193, 2011.

19. Gurgel RQ, Cardoso GS, Silva AM, Santos L.N; Oliveira RCV. Creche: ambiente expositor ou protetor nas infestações por parasitas intestinais em Aracaju, SE. Rev Soc Bras Med Trop 38: 267-269, 2005.

20. IBGE. Censo demográfico: 2010. Rio de Janeiro: IBGE. Disponível em: $<$ http://www.ibge.gov. br/home/estatistica/populacao/censo2010/aglomerados_subnormais/agsn2010.pdf > . Acesso em: 20/10/2012.

21. Komagome SH, Romagnoli MPM, Previdelli ITS, Falavigna DLM, Dias MLGG, Gomes LM. Fatores de risco para infecção parasitária intestinal em crianças e funcionários de creche. Cienc Cuid Saúde 6: 442-447, 2007.

22. Lutz AO. Schistosomum mansoni, segundo observações feitas no Brasil. Mem Inst Oswaldo Cruz 11: 121-155, 1919.

23. Mariath AB, Giachini RM, Lauda LG, Grillo LP. Estado de ferro e retinol sérico entre crianças e adolescentes atendidos por equipe da Estratégia de Saúde da Família de Itajaí, Santa Catarina. Cienc Saúde Colet 15: 509-516, 2010.

24. Mariz ICL, Nóbrega MFF. Enteroparasitoses em crianças de 0 a 6 anos atendidas em um Hospital Infantil de Campina Grande, PB. Rev Newslab 95: 104-109, 2009. 
25. Penna HAO, Oselka GW. Doenças infecto-contagiosas e parasitárias em Pediatria. In: Marcondes E. Pediatria Básica. Sarvier. São Paulo, 1978.

26. Pezzani B, Ciarmela ML, Apezteguía MC, Molina N, Orden A, Rosa D, Minvielle M. Intestinal parasitoses in suburban and rural schoolchildren in Argentina. Rev Patol Trop 41: 63-73, 2012.

27. Rey L. Parasitologia: Parasitos e doenças parasitárias do homem nas Américas e na África. Guanabara Koogan. Rio de Janeiro, 2008.

28. Santos HLC, Martins LAF, Peralta RHS, Peralta JM, Macedo HW. Frequency of amoebiasis and other intestinal parasitoses in a settlement in Ilhéus City, State of Bahia, Brazil. Rev Soc Bras Med Trop 47: 101-104, 2014.

29. Saturnino ACRD, Marinho EJC, Nunes JFL, Silva EMA. Enteroparasitoses em escolares de $1^{\circ}$ grau da rede pública da cidade de Natal, RN. Rev Bras Anal Clin 37: 83-85, 2005.

30. Seixas MTL, Souza JN, Souza RP, Teixeira MCA, Soares NM. Avaliação da frequência de parasitos intestinais e do estado nutricional em escolares de uma área periurbana de Salvador, Bahia, Brasil. Rev Patol Trop 40: 304-314, 2011.

31. Silva EF, Silva EB, Almeida KS, Souza JJN, Freitas FLC. Enteroparasitoses em crianças de áreas rurais do município de Coari, Amazonas, Brasil. Rev Patol Trop 38: 35-43, 2009.

32. Silva TV, Leda LR. Intervenções educativas sobre parasitoses intestinais: aplicação de um jogo para alunos do ensino fundamental. Saúde \& Amb Rev 7: 23-37, 2013.

33. Sogayar MITL, Guimarães S. Giardia. In: Neves DP, Melo AL, Genaro O, Linardi PM. Parasitologia humana. Atheneu. Rio de Janeiro, 2005.

34. Tan KSW. New insights on classification, identification, and clinical relevance of Blastocystis spp. Clin Microbiol Rev 21: 639-665, 2008.

35. Teixeira ML, Flores RE, Fuentefria AM. Prevalência de enteroparasitas em crianças de uma creche na cidade de Concórdia, Santa Catarina, Brasil. Rev NewsLab 78: 110-116, 2006.

36. Uchôa CMA, Albuquerque MC, De Carvalho F.M. Parasitismo intestinal em crianças e funcionários de creches comunitárias na cidade de Niterói-RJ, Brasil. Rev Patol Trop 38: 267-278, 2009.

37. Vargas M, Gascon J, Casals C. Etiology of diarrhea in children less than five years of age in Ifakara, Tanzania. Am J Trop Med Hyg 70: 536-539, 2004.

38. Vasconcelos IAB, Oliveira JW, Cabral FRF, Coutinho HDM, Menezes IRA. Prevalência de parasitoses intestinais entre crianças de 4-12 anos no Crato, Estado do Ceará: um problema recorrente de saúde pública. Acta Sci Health Sci 33: 35-41, 2011.

39. Zaiden MF, Santos BMO, Cano MAT, Nascif-Júnior IA. Epidemiologia das parasitoses intestinais em crianças de creches de Rio Verde - GO. Medicina (Ribeirão Preto) 41: 182-187, 2008. 